\title{
Hüftgelenkdysplasie beim Hund - Chancen und Risiken
}

\author{
Andreas Zohmann, Carsten Grußendorf, Hanna Diekmann, Veit Kleine Büning
}

In Deutschland leiden rund 250000 Hunde an Hüftgelenkdysplasie (HD). Je früher man die Krankheit erkennt und tätig wird, desto größer ist die Chance, Folgeschäden zu verhindern und ein weitgehend schmerzfreies Leben zu ermöglichen. Mit Physiotherapie können sowohl prophylaktisch unterstützend als auch postoperativ gute Erfolge erzielt werden. Doch welche physiotherapeutischen Maßnahmen sind wann sinnvoll? Was ist postoperativ zu beachten?

\section{Definition}

Die HD ist eine der bedeutendsten orthopädischen Erkrankungen des Hundes mit ausgeprägtem genetischen Hintergrund. Es ist eine konstruktive Gelenkerkrankung, deren phänotypisches Erscheinungsbild nur in geringem Maße von Umweltfaktoren beeinflusst wird. Neben der fehlerhaften Anlage von Acetabulum, Femurkopf und der weichen Gelenkanteile kann auch deren Entwicklung und Ausbildung betroffen sein. Der Verlauf ist progressiv und mündet immer in einer mehr oder weniger ausgeprägten Osteoarthrose.

\section{Diagnostik beim adulten Hund}

\section{Adspektion, Palpation und Gelenkfunktionsprüfung}

Untersuchung des Hundes:

- Gangbildanalyse an kurzer Leine im Schritt:

- Schwingen des Beckens: Der LSÜ-Twist (lumbosakraler Übergangstwist) sollte nach der knöchernen Ausbildung der Hüftpfanne, dem Ausreifen des Hüftgelenks in der 20. Lebenswoche, abklingen.

- Bleibt der LSÜ-Twist weiterhin bestehen oder verstärkt sich noch, ist dies ein starker Hinweis auf eine Schonung des Hüftgelenks.

- Adspektion in der Ruhe:

- Von hinten erkennbare Atrophie der Glutealmuskulatur sowie des M. biceps femoris mehr oder weniger ein- bzw. beidseits.

- Im Sitz nicht selten Körperverlagerung auf die schmerzhaftere Gesäßhälfte (cave: keine Verwechslung mit dem sog. „Kniesitz“ nach Kreuzbandriss!).
- Palpation gelenkspezifischer Triggerpunkte, speziell des sog. proximalen Oberschenkel-Triggerpunkts (Punkt MA31 der Akupunktur, $>$ Abb. 1):

- Der nahe des Hüftgelenks im M. rectus femoris liegende Triggerpunkt steht bei einer Problematik dieses Gelenks unter erhöhtem Tonus.

- Druck auf diesen Triggerpunkt veranlasst den Hund zu einer Ausweich-, Schmerz- oder Abwehrreaktion.

- Gelenkfunktionsprüfung:

- Das Hüftgelenk wird in Flexion, Extension und Abduktion geprüft. Einschränkungen bis Schmerzhaftigkeiten ergeben sich v.a. durch erhöhte Spannung der Glutealmuskulatur (in Flexion), M. iliopsoas (Extension) und/oder M. pectineus (Abduktion).

- Die Rotationstestung ist teilweise sehr schmerzhaft und sollte unseres Erachtens vermieden werden.

- Ortolani-Test: Dient der Überprüfung der Straffheit der Gelenkkapsel. Bei Laxizität derselben spricht man von einer Weichteil-HD oder „lockeren Hüfte“.

\section{ORTOLANI-TEST}

Cave: Der Ortolani-Test kann schmerzhaft sein und ist meistens nur in Sedation aussagekräftig, da dann die Muskulatur komplett relaxiert ist. Wir halten den Test für Hunde < 5 Monaten nicht sinnvoll, da das Hüftgelenk zu diesem Zeitpunkt noch nicht ausgereift ist. 


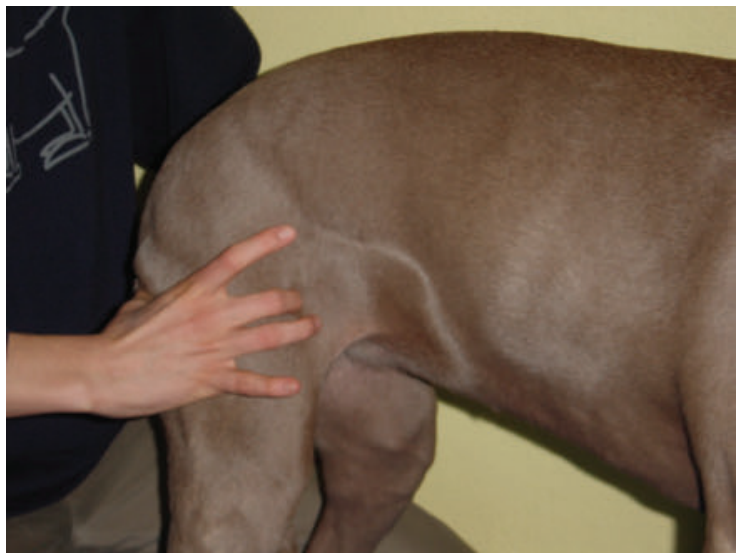

- Abb. 1 Palpation des MA31: am kranialen Rand des Oberschenkels, am Rand des obersten Drittels zwischen Patella und Tuber coxae, zwischen M. sartorius und M. tensor fasciae latae. ( ) Vierbeiner Reha-Zentrum GmbH

\section{Röntgenologisch}

\section{Beurteilt werden:}

- Gelenkpfanne (Pfannendachkontur): physiologisch gerade oder sanft s-förmig

- kraniale Pfannenkontur: konkret oder etwas „verwaschen“

- kraniolateraler Pfannenrand: mit dem Femurkopf abschließend oder nicht

- Femurkopf: Form und Kongruenz mit dem Pfannenboden

- Femurhals: noch schlank oder arthrotische Zubildungen, Morgan-Linie?

- Gelenkspalt

- Position des Femurkopfmittelpunkts soll sich innerhalb des Pfannendachrands befinden

- Norbergwinkel ( $>$ Abb.2): physiologischer Wert liegt bei $>105^{\circ}$

- Folgeerscheinungen: arthrotische Zubildungen (Coxarthrose)?

\section{Frühdiagnostik}

Die Früherkennung der HD des Hundes zählt nach wie vor zu den besonderen Herausforderungen der modernen orthopädischen Diagnostik. Angestrebt wird eine möglichst zweifelsfreie Bestimmung des Zustands der Hüftgelenke beim Welpen und/oder Junghund mit dem bei der Screening-Untersuchung mit Zuchtzulassung oder Gebrauchstauglichkeit zu rechnen sein wird.

\section{Adspektion, Palpation und Gelenkfunktionsprüfungen}

- Auffälligkeiten im freien Verhalten des Junghunds, z. B. im Spiel mit anderen:

- häufiges Hinsetzen

- Nachbellen

\section{- Aggressivität bei Körperkontakt}

- Gangbildanalyse

- Adspektion in der Ruhe

- Palpation

- Gelenkfunktionsprüfungen und die Testung nach Ortolani auf Weichteil-HD (lockere Hüfte)

Gangbildanalyse, Adspektion, Palpation und Gelenkfunktionsprüfungen erfolgen wie in der Diagnostik des adulten Hundes beschrieben.

\section{Röntgenologisch}

\section{PennHIP ${ }^{\circledR}$-Methode nach Smith}

PennHIP (Pennsylvania Hip Improvement Program) ist ein offizielles Programm zur Früherkennung der HD bei Hunden ab der 16. Lebenswoche. Grundlage dieser Untersuchung ist die Erkenntnis, dass eine sog. Weichteil-HD maßgeblich durch die Laxizität der Hüftgelenkkapsel verursacht wird.

Bei dieser sog. „Stress-Untersuchung“ (in Sedation) werden die Femurköpfe einem von medial nach lateral wirkenden Druck ausgesetzt. Das heißt, es wird versucht, sie aus der Pfanne zu drücken, sofern dies aufgrund der „joint laxity“ möglich ist. Die PennHIP-Methode nach Smith ist ein Verfahren mit einem Hilfsmittel (Distraktor), sodass sowohl eine Kompression des Femurkopfs in

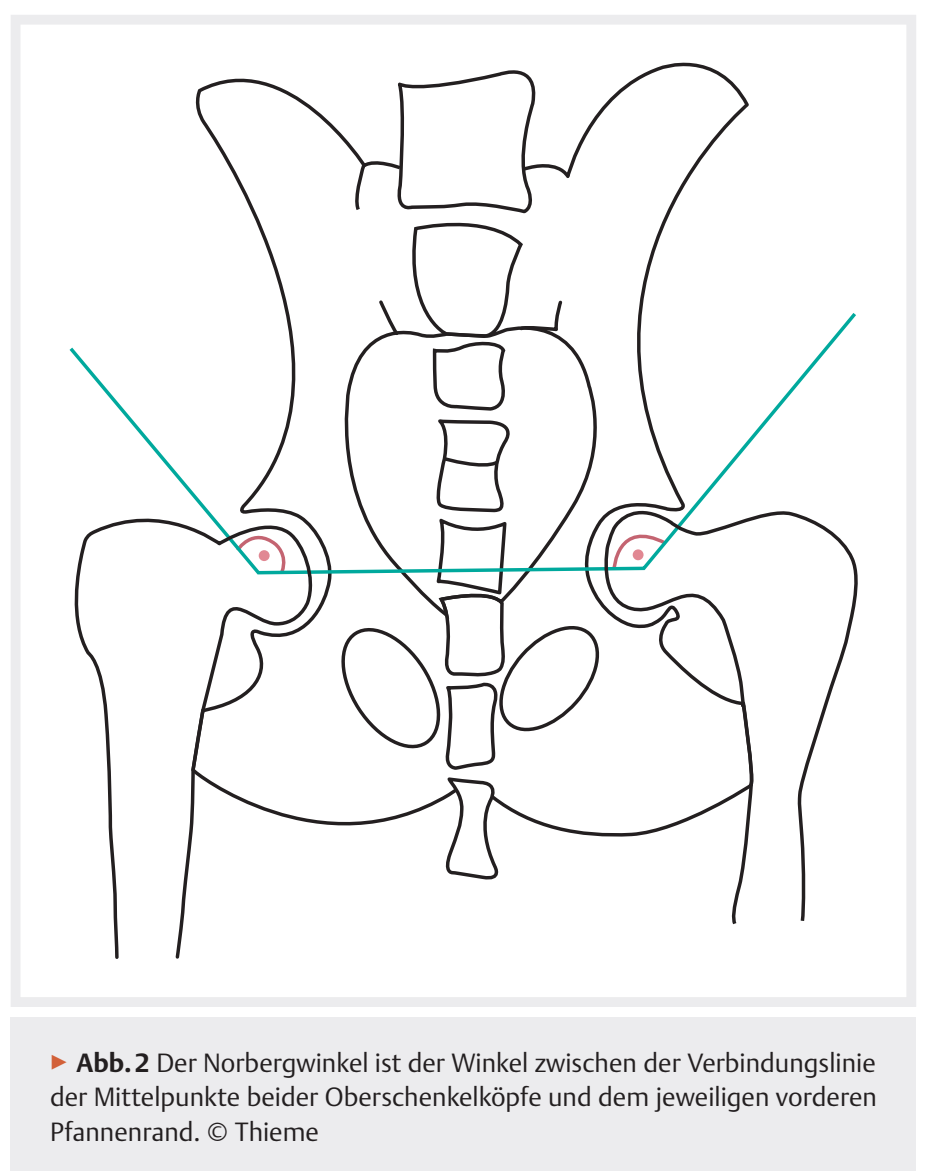


die Pfanne als auch deren maximale Distraktion nach lateral ermöglicht wird. Der Distraktionsindex wird als Maß für die Lockerheit des Gelenks angegeben. Daraus wird der zu erwartende Grad der HD abgeleitet.

Dieses spezielle Röntgenverfahren ist zertifiziert und darf nur von speziell ausgebildeten und geschulten Tierärzten durchgeführt werden. Die Aufnahmen werden immer offiziell vom PennHIP-Center der University of Pennsylvania ausgewertet.

\section{Die anatomisch-morphologische Beurteilung nach Köppel}

Beurteilt wird hierbei das heranwachsende Hüftgelenk im Alter zwischen der 16. und 20. Lebenswoche. Aufgrund der Verknöcherungsvorgänge an der Hüftgelenkpfanne wird auf den möglichen Grad der HD geschlossen.

Bei der anatomisch-morphologischen Beurteilung werden im Röntgenbild das Os acetabulare und das Os coxae quartum (gleicht in seiner Form einem auf der Seite liegenden „T“) beurteilt. Diese beiden primären Ossifikationszentren sind form- und konturgebend für das Pfannendach und auch für die Tiefe des Acetabulums.

Wichtige Fakten:

- Mit dem Ende der 20. Lebenswoche hat das Acetabulum seine endgültige Form erreicht.

- Störungen der Entwicklung bleiben zeitlebens bestehen und führen zu charakteristischen Formen des Acetabulumdachs.

- Nach diesem Zeitpunkt auftretende Formänderungen sind sekundär und in vielen Fällen als Instabilitätsmerkmale zu interpretieren.

Hypoplasien, Aplasien und Persistenzen dieser Ossifikationskerne lassen sich im Röntgenbild nachweisen und können als Hinweise für die Diagnose HD angesehen werden.

\section{Folgeschäden der HD und Schadensbegrenzung}

Die HD kann viele Folgeschäden verursachen:

- Die Fehlstellung des Kniegelenks führt zur Überlastung, v. a. des vorderen Kreuzbands.

- Entlastungs- bzw. Ausweichbewegungen belasten die Sakroiliakalgelenke sowie den lumbosakralen Übergang (Cauda equina).

- Lastübernahmen von hinten nach vorne ziehen Probleme im Ellbogen, Bizeps- und Trizepsbereich und besonders der Vorderzehengrundgelenke sowie deren proximaler Interphalangealgelenke nach sich.
Sehr häufig sind es dann Hunde mit gerade diesen Problemen, die zur Chirurgie und/oder Physiotherapie vorgestellt werden. Leider hat es sich noch immer zu wenig herumgesprochen, dass die angeführten Pathologien Alarmsignale darstellen, die unbedingt eine Überprüfung der Hüftsituation bedingen sollten. Gut beraten ist dann, wer nach der eigentlichen Ursache fahndet und das Hüftgelenk intensiv mitbehandelt: „Es schreit immer das Opfer, nie der Täter!“

Merke

Früherkennung ist wichtig, um Folgeschäden zu vermeiden.

Dies beginnt für den prospektiven Hundebesitzer schon bei der Wahl der Hunderasse: Es sind nun einmal wunderbare Rassen, die aufgrund ihrer Beliebtheit leider - sagen wir es diplomatisch - „nachlässig“ gezüchtet werden und mangelhafte Hüftzustände aufweisen, z.B. :

- Deutscher Schäferhund

- Golden Retriever

- Labrador

- Berner Sennenhund und viele andere mehr

Die berühmte Entscheidung für einen Mischling ist schon lange keine Garantie mehr für Hüftgesundheit. Wichtig ist die sorgfältige Beobachtung von Spielverhalten und Gangbild, die bei Auffälligkeiten eine Überprüfung durch den Tierarzt erfordern. Dabei kommt entsprechend ausgebildeten Hundetrainern und Tierphysiotherapeuten die wichtige Rolle des „missing link“ zwischen Hundebesitzer und Tierarzt zu. Der geschulte Blick des Profis erkennt auch das, was der Hundehalter als eigentlich völlig normal ansieht.

\section{Konservative oder chirurgische Behandlung?}

Die erste Frage, die sich der Tierarzt oder Tiertherapeut zu stellen hat: Erlaubt dieses Hüftgelenk eine rein konservative Behandlung oder ist eine stabilisierende oder aber palliative Chirurgie von Nöten? Wie beantwortet sich diese Frage?

Die Entscheidung sollte anhand des folgenden Prinzips getroffen werden:

- Wie stellt sich die anatomisch-konstitutionelle Situation dar und wie ist der konditionelle Zustand des Patienten?

- Bei manchen Rassen bzw. Zuchtlinien sind die Hüft-, Knie- und Tarsalgelenke stark gewinkelt (Deutscher Schäferhund bis zu $90^{\circ} / 90^{\circ} / 90^{\circ}$ ). Wenn nun die hüftgelenkstabilisierende Muskulatur (v. a. M. gluteus medius und M. biceps femoris) bereits fortgeschritten atrophiert ist, ist die 
Prognose für eine rein konservative Behandlung nicht günstig.

- Steiler gestellte Gelenke (natürlich keine Stuhlbeinig- oder sogar Vorbiegigkeit!) vergesellschaftet mit einer guten Tendenz, Muskulatur aufzubauen (z. B. beim Rottweiler) bieten hier für einen konservativen Therapiezugang eine prognostisch günstigere Ausgangslage. Auch wenn es gilt, eine dann doch noch notwendige Chirurgie hinauszuzögern.

- Wie sieht der radiologische Befund bei der Rasse, in dem Alter und bei dem Gewicht aus?

- Ein Beispiel: Ein schlanker, junger Rottweiler hat bei einer diagnostizierten HD eine weitaus bessere Prognose für die konservative physiotherapeutische Behandlung im Sinne eines hüftstabilisierenden Muskelaufbaus als der gleichalte und ebenso schwere Schäferhund. Bei Letzterem ist auch noch zu beachten, dass die Winkelungen der Hinterbeingelenke Einfluss auf die Dynamik des lumbosakralen Übergangs im Sinne einer zunehmenden Hypermobilität nehmen können. Die Folge ist eine zunehmende Beeinträchtigung des Cauda-equinaBereichs.

- Und die über allem stehende Frage: Wie steht es um die Schmerzsituation dieses Hundes?

- Denn es gilt: Keine Physiotherapie unter Schmerz! Hier ist abzuwägen, ob eine begleitende Schmerztherapie (mit oder ohne Medikation, z. B. mit Akupunktur) zur Physiotherapie am sinnvollsten ist und in zielführendem Maße einbezogen werden soll oder ob diese auch für sich schon schmerzreduzierend sein kann.

\section{SCHMERZTHERAPIE}

Die Schmerztherapie als Begleitung bzw. Ermöglichung einer sinnvollen Physiotherapie wird als eigenes Thema in einer der nächsten Ausgaben der „Hands on“ näher behandelt.

\section{Physiotherapie und physikalische Therapie}

Für die Auswahl der geeigneten Methoden und Übungen müssen wir folgende Unterscheidungen vornehmen:

- HD nach Früherkennung (innerhalb der 1. Lebensjahreshälfte)

- HD diagnostiziert anlässlich der „klassischen“ HD-Diagnostik (mit 1 Jahr oder nach ca. 11/2 Jahren, je nach Rassengröße)

- HD-Erkennung nach längerer Zeit

- Zufallsbefund (bei radiologischer Untersuchung mit anderer Indikation)

- „klinisch inapparente HD“ (bis jetzt nicht vordergründig auffällige HD)
- als mögliche Ursache für Probleme des gesamten Bewegungsapparats

- Status nach Hüftoperation

\section{HD nach Früherkennung}

Die HD wurde innerhalb des 1. Lebensjahrs-idealerweise zwischen der 16. und 20. Lebenswoche-diagnostiziert. Die Prognose für den Muskelaufbau ist gut (siehe: Konservativ oder chirurgisch?).

Empfehlenswert sind die folgenden Maßnahmen:

\section{Aktive Bewegungstherapie}

Jede der 3 folgenden Übungen jeweils 5 Minuten/Tag über den Tag verteilt am Brustgeschirr oder an kurzer Leine im Schritt durchführen.

- Slalom

- 4-5 Stangen, ca. 1,5 Hundelängen Abstand

- Training der Adduktoren und Abduktoren

- Cavaletti

- 4-5 Hürden so hoch bzw. niedrig, dass der Hund sie übersteigt, nicht überspringt

- Abstand so, dass jeweils nur Platz für 1 Pfotenstep ist, der Hund also bei jedem Schritt abwechselnd mit Hinter-, aber auch Vorderbeinen darübersteigt $(\triangleright$ Abb. 3)

- v.a. Kräftigung des M. rectus femoris, des M. iliopsoas (Hüftbeuger) sowie der Glutealmuskulatur (Hüftstrecker)

- Bergauf gehen

- 10 - 15\%ige Steigung

- der Hund darf ruhig gegen die Leine ziehen!

- v. a. Kräftigung der Beckenstrecker (Mm. glutei) sowie durch verstärkte Abduktion der Hinterextremitäten Kräftigung des M. biceps femoris

- ideale Übung zur Stabilisierung des Hüftgelenks

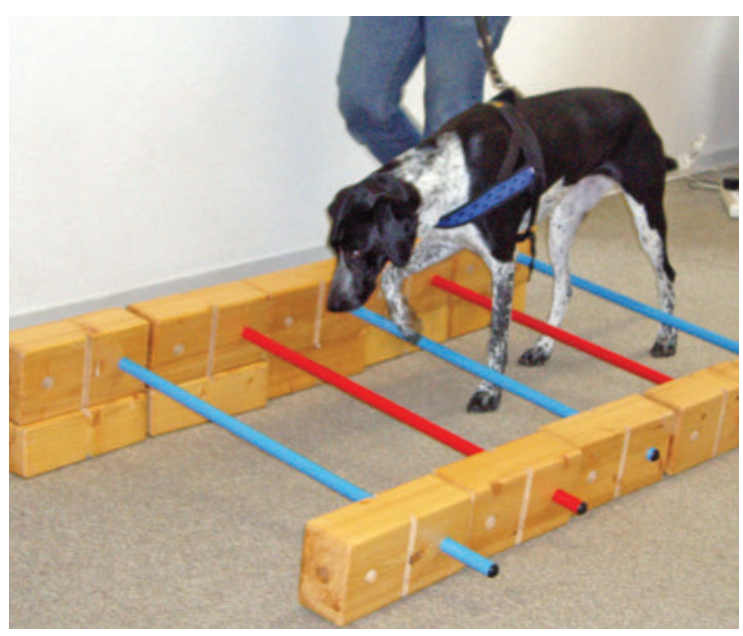

- Abb. 3 Beim Cavaletti-Training sollte der Hund im Schritt über die Stangen steigen und nicht darüber springen. @ Vierbeiner Reha-Zentrum GmbH 


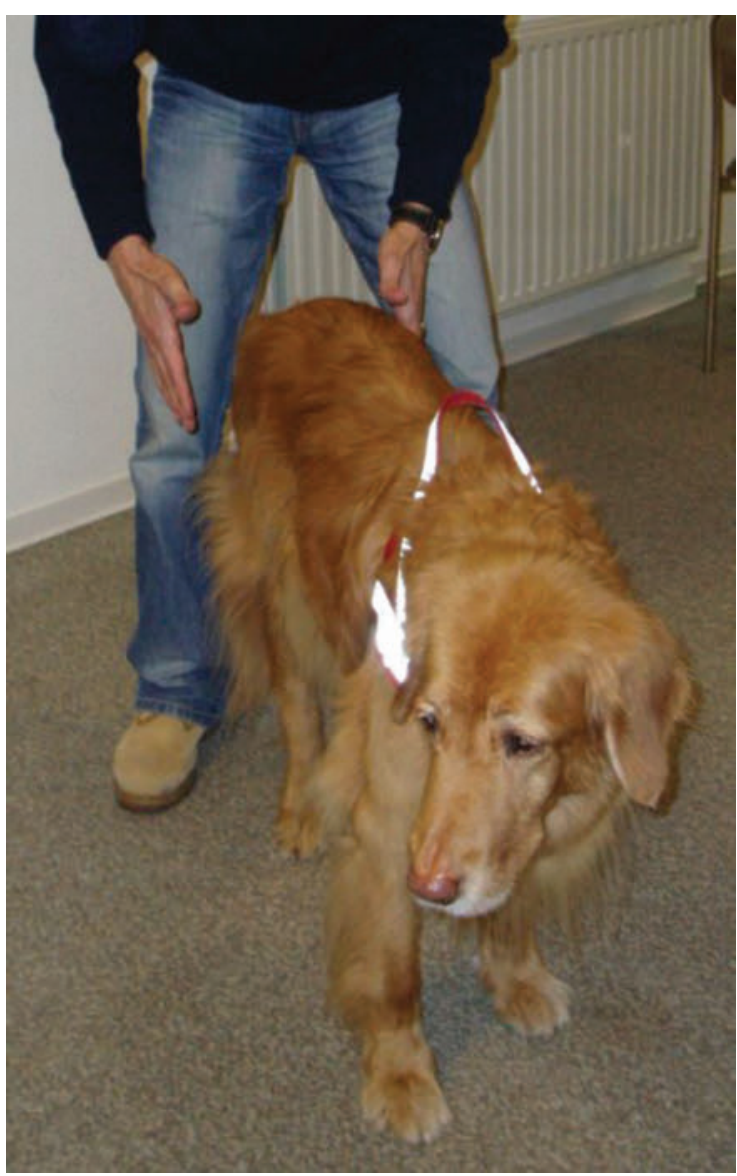

- Abb.4 Die Hände erzeugen einen wechselseitigen Druck seitlich gegen das Becken. @ Vierbeiner Reha-Zentrum $\mathrm{GmbH}$

\section{Aktiv-assistive Bewegungstherapie}

Die „isometrischen Übungen“ können mehrfach am Tag durchgeführt werden:

- beide Hände seitlich im Bereich des Beckens flach auflegen, durch wechselseitigen Druck gegen das Becken Gewicht von einer Seite auf die andere verlagern ( $\triangleright$ Abb.4), ohne dass der Hund durch einen Seitenschritt ausgleichen muss (entspricht in der aktiven Bewegungstherapie dem Slalom)

- beidseits Handflächen vorne an die Oberschenkel legen und mit so viel bzw. wenig Kraft nach hinten auf sich zu ziehen, dass der Hund noch stehen bleibt und Widerstand gibt ( $\triangleright$ Abb.5)

- beide Hände flach übereinander auf den Kreuzbereich auflegen ( $\triangleright$ Abb.6) und mit mehrfach aufeinander folgendem leichten Druck den Beckengürtel wippend nach unten drücken, der Hund bleibt dabei stehen und drückt immer wieder nach oben (entspricht in der aktiven Physiotherapie der Cavaletti-Übung)

- eine Hand im Bereich des Beckens, die andere am kontralateralen Schulterblatt flach auflegen ( $\mathbf{A} \mathbf{A b} \mathbf{b} . \mathbf{7})$ und gleichzeitig wippenden Druck ausüben, dann die Handgriffe diagonal wechseln, kräftigt die

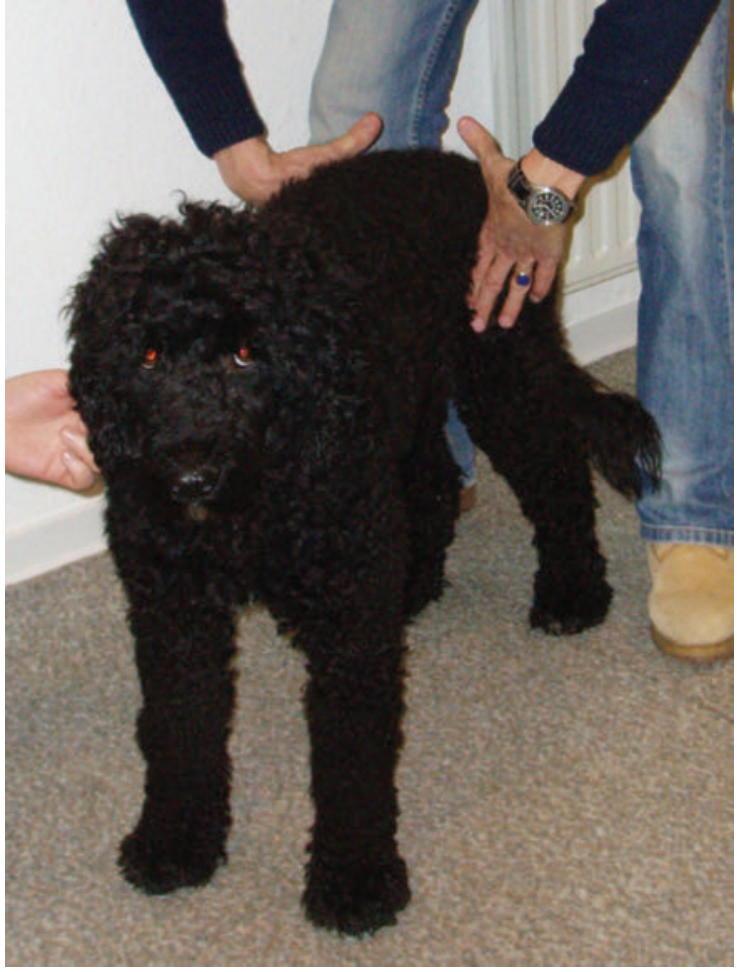

- Abb. 5 Die Hände von vorne an die Oberschenkel legen und leicht nach hinten drücken, sodass der Hund stehen bleibt, aber Widerstand gibt. @ Vierbeiner Reha-Zentrum $\mathrm{GmbH}$

autochthone Muskulatur und stabilisiert damit den lumbosakralen Übergang (LSÜ)

\section{Gleichgewichtsübungen}

Diese Methoden lassen sich noch durch Gleichgewichtsübungen auf dem Schaukelbrett, einem Trampolin oder einer nicht gänzlich aufgepumpten Luftmatratze (der Phantasie und weiteren Kreativität ist dahingehend keine Grenze gesetzt) optimieren.

\section{Unterwasserlaufband}

Das Gehen bzw. Laufen im Wasser (Wasserspiegel auf Niveau der Rumpfmitte) ist dem Schwimmen vorzuziehen. Die Mehrzahl der Hunde tritt beim Schwimmen mit den Hinterextremitäten nach unten, um sich an der Wasseroberfläche zu halten, während die Vorderbeine durch Kraulbewegungen das Vorwärtskommen bewerkstelligen. Diese steppende (nach unten tretende) Bewegung der Hinterbeine trainiert aber in erster Linie den beim Mensch kräftigsten Gesäßmuskel (M. gluteus supferficialis sive maximus), der beim Vierbeiner nicht den kräftigsten Hüftstabilisator repräsentiert. Hier ist es der $\mathrm{M}$. gluteus medius mit seinem schrägen Faserverlauf und dieser wird nicht durch eine nach unten steppende Beinbewegung trainiert, sondern durch das Gehen im Wasser wie auch durch das Bergaufgehen. Weiterhin bringt der im Wasser gehende Hund seine Beine vermehrt in Abduktion, was zur Kräftigung des M. biceps femoris führt-also des 2. starken Hüftstabilisators. 


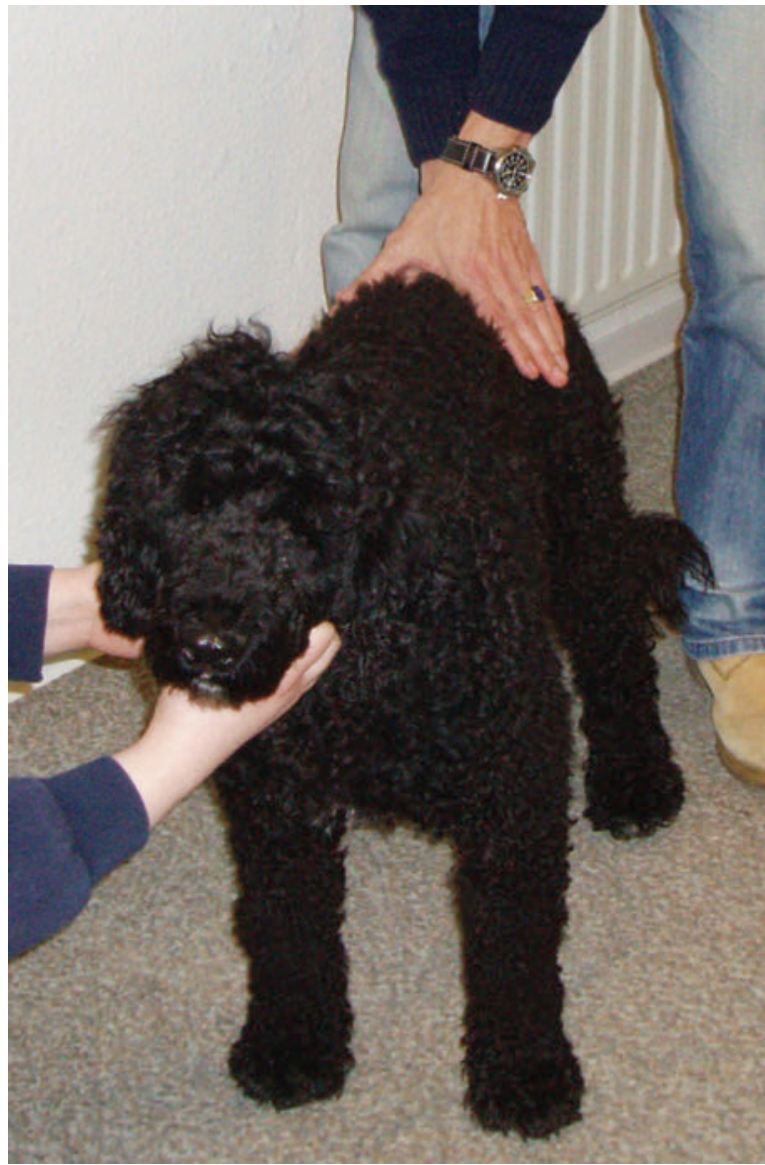

- Abb. 6 Hände übereinander flach auf das Kreuzbein legen und den Beckengürtel leicht wippend nach unten drücken. (c) Vierbeiner Reha-Zentrum GmbH

\section{HD bei Hunden > 1,5 Jahren}

Nun lebt der Hund schon längere Zeit mit einer zumindest unangenehme Empfindungen auslösenden Hüfte. Die Folge sind Atrophien der eben genannten Muskeln und stärkere Gangbildveränderungen.

Zu den oben beschriebenen Methoden kommen nun zusätzlich zum Einsatz:

\section{Massage}

Tonisierende Massagetechniken wie rasche Streichungen, Knetungen und Schüttelungen speziell der atrophierten Muskulatur sind empfehlenswert.

- Tapping (engl. to tap=klopfen, pochen): Diese spezielle Form der Klopfung (des Tapotements, frz.) geschieht derart, dass man nur mit den Fingerkuppen einige Minuten lang kräftige und dennoch leichte Bewegungen ausübt. Die Technik ist leicht, aber stabil, weil immer dieselbe Stelle getroffen wird. Die anzuwendende Kraft ist daher auch nicht groß, doch müssen die Bewegungen rasch, elastisch federnd und ganz aus dem lockeren Handgelenk kommen. Die Fingerkuppen federn quasi von der Muskulatur des Tieres zurück - beim Hund mit HD betrifft dies v. a. die

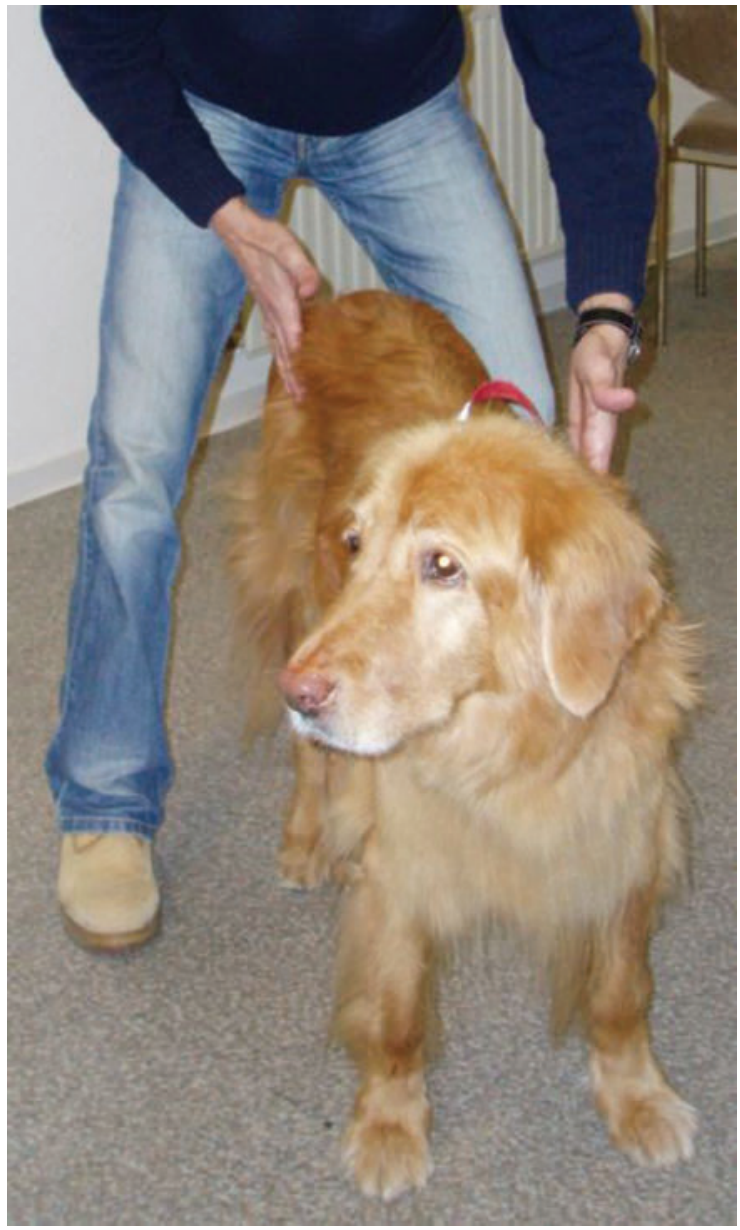

- Abb. 7 Eine Hand seitlich flach an das Becken, die andere an das kontralaterale Schulterblatt legen und gleichzeitig gegeneinander wippenden Druck ausüben. (c) Vierbeiner Reha-Zentrum $\mathrm{GmbH}$

von Atrophie bedrohte oder schon atrophe Glutealmuskulatur sowie den M. biceps femoris mit dem Ziel einer forcierten Tonussteigerung.

- Zwischen den einzelnen Massagetechniken dürfen vorübergehend detonisierende Streichungen nicht fehlen, um einen etwaigen Hypertonus zu vermeiden.

\section{Aktiv-assistive Bewegungstherapie}

Einsatz leichter bzw. mittelleichter Gewichtsmanschetten. Je nach Größe und Kondition des Patienten genügen zumeist 100-250 g. Die Manschette stört den Hund und er wird versuchen, dagegen anzuarbeiten.

\section{- Training mit Gewichten:}

- Eine Manschette unterhalb des Tarsus führt meistens zum Anziehen (Beugen) der gesamten Hinterextremität, also zur Aktivierung des M. rectus femoris, M. iliopsoas und der Anteile der „ham strings“ (M. biceps femoris, M. semitendinosus, M. semimembranosus) sowie der Strecker des Unterschenkels. 
- Manschette oberhalb des Tarsus führt in vielen Fällen zu einer Streckung im Hüftgelenk, also Aktivierung der Glutealmuskulatur sowie Anteile der „ham strings“ (M. biceps femoris, M. semitendinosus, M. semimembranosus) sowie der kruralen Beuger (v. a. M. gastrocnemius).

- Da dieser Bewegungsablauf reflexartigen Charakter hat und unkontrolliert abläuft, ist vor der Bewegungsdurchführung zu überlegen, ob dies für den jeweiligen Patienten die richtige Trainingsform darstellt. Und: Nicht jeder Hund reagiert auf diese Stimuli in gleicher Form. Daher cave und testen!

- $\mathrm{MOBI}^{\odot}$ - musterorientierte Bewegungsinduktion:

- Stimulation des M. biceps femoris nach Fuchs: Der $M$. biceps femoris hat eine hüftgelenkstabilisierende Funktion. Eine Aktivitätsanregung des Muskels erreicht man durch eine aktiv-assistive Technik, die ursprünglich aus der propriozeptiven neuromuskulären Fazilitation (PNF) hergeleitet wurde. Dabei wird die Hinterextremität des in Seitenlage befindlichen Tieres in Hüftextension, -abduktion und -innenrotation gebracht sowie das Knie flektiert.

- Dann erfolgt der Stimulus gegen die Pronation des Fußes (durch Kneifen mit den Fingern in die Zwischenzehenhaut wie bei der Testung des Flexorreflexes) und als Reaktion darauf kann die Kontraktion des M. biceps femoris fühl- und sichtbar hervorgerufen werden. Detaillierte Informationen finden Sie im Beitrag „MOBI beim Hund“.

- Reflexinduziertes Training nach Alexander (RITA):

- Hierbei werden polysynaptische Reflexe (sog. „Massenreflexe“) genutzt, um die Muskulatur zu kräftigen. In erster Linie verwenden wir den Flexorreflex an der Hinterextremität, der zwar nur den N. tibialis anspricht, dennoch unterschiedliche Reaktionen der Beckengliedmaße nach sich ziehen kann.

- Den Zwischenzehenreflex durch Kneifen der Zwischenzehenhaut auslösen, aber dabei mit den Fingern nicht loslassen - der Hund zieht gegen unseren gehaltenen Widerstand! Je nachdem, welche Muskulatur gekräftigt werden soll, ist auszutesten, ob als Ausgangsposition des Hinterbeins die Normal-, Retraktions- oder die Protraktionsstellung gewählt wird. Achtung, nicht jeder Hund zeigt die gleiche Reaktion (siehe Training mit Gewichten)!

\section{Nach Hüftoperation}

Die Physiotherapie kann nach jeder OP-Methode (sofort) eingesetzt werden. Natürlich ist die Wahl der Therapieformen von der jeweiligen chirurgischen Intervention abhängig. Die Palette reicht von minimalsten Stimuli wie manueller Lymphdrainage, Thermotherapie und einer speziellen Form der Elektrotherapie, der Interferenzstrom-
Regulationstherapie (IFR ${ }^{\odot}$ ) sowie vorsichtig ausgeführten „isometrischen“ Übungen bis hin zu rasch einsetzender intensiverer aktiver Physiotherapie. Prinzipiell sind alle der bisher beschriebenen Methoden für den Patienten adäquat.

\section{PRAXISTIPP}

Zu welchem Zeitpunkt welche postoperative Therapieform in Abhängigkeit von der gewählten OPMethode eingesetzt werden kann, sollte immer mit dem Chirurgen besprochen werden.

Es muss abgewogen werden, ob es sich beispielsweise um ...

- einen minimal invasiven Eingriff (z. B. Goldimplantation),

- einen mittelgradig invasiven Eingriff (z. B. Femurkopfhalsresektion) oder

- einen hoch invasiven Eingriff (z. B. Umstellungsosteotomie, zementierte oder nicht zementierte Totalendoprothese) handelt.

Anders als bei den anderen beschriebenen OPMethoden ist es bei der Femurkopfhalsresektion ausdrücklich erwünscht, dass sich der Patient nach der Operation frühzeitg intensiv bewegt, um einen stimulierenden Impuls zu geben. Nach eigenen Erfahrungen lassen sich die Erfolgsergebnisse durch intensive und frühzeitige Physiotherapie deutlich verbessern.

In den meisten Fällen können folgende Methoden schon am 1. postoperativen Tag durchgeführt werden:

- manuelle Lymphdrainage

- Interferenzstrom-Regulationstherapie-IFR (Achtung, nicht zu verwechseln mit herkömmlichem Interferenzstrom!)

- aktiv-assistive Methoden (wie unter HD nach Früherkennung und bei Hunden > 1,5 Jahren beschrieben)

\section{EXKURS: CHIRURGISCHE MAßNAHMEN}

Bei der chirurgischen Versorgung der HD unterscheidet man je nach Vorgehen zwischen prophylaktischen, palliativen oder wiederherstellenden Maßnahmen. Bei prophylaktischen Eingriffen wie der juvenilen pubischen Symphysiodese wird versucht, frühzeitig diagnostizierte Patienten vor der Entwicklung von sekundären Arthrosen zu bewahren. Bei Methoden wie der Totalendoprothese oder der Femurkopfhalsresektion wird versucht, das Hüftgelenk zu ersetzen, um so die Schmerzen zu eliminieren und eine angemessene Funktion wiederherzustellen. Palliative Möglichkeiten wie eine 
Goldimplantation, Myotomie oder eine Denervation versuchen, eine geeignete Schmerzlinderung herbeizuführen.

Die jeweiligen Therapieoptionen hängen von folgenden Faktoren ab:

- Alter des Patienten

- aktueller Zustand der Hüfte

- Wahrnehmung und Erwartungshaltung der Besitzer

(Die häufigsten chirurgischen Maßnahmen bei HD werden in einem Beitrag in einer der kommenden Ausgaben der Hands on noch ausführlicher erläutert.)

\section{Take home}

In Zeiten des digitalen Röntgens hat sich die Diagnostik und Früherkennung der HD beim Hund deutlich verbessert. Durch verschiedene Untersuchungen und Röntgenaufnahmen ist es möglich, erkrankten Patienten eine ideale Versorgung zu bieten. Die Therapie richtet sich in den meisten Fällen nach Alter des Patienten, Schweregrad der Erkrankung, Erwartungen der Besitzer sowie Kostenaufwand. Der wichtigste Bestandteil bei der Wahl der jeweiligen Intervention (sei es OP oder konservativer Therapieversuch mithilfe von Entzündungshemmern und Physiotherapie oder wahlweise der Kombination aus beidem) sollte von der klinisch-orthopädischen Untersuchung abhängig gemacht werden. Um feststellen zu können, wie stark ein Hund durch die HD eingeschränkt wird, sollte nicht allein eine Röntgenaufnahme hinzugezogen werden, sondern immer der klinische Zustand bei Bewegung der Hüften entscheidend sein.

\section{Autoren}

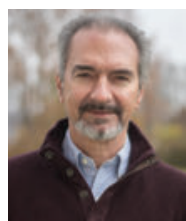

\section{Andreas Zohmann}

Dr. med. vet.; Leitender Tierarzt des Vierbeiner-Rehazentrums; fachlicher Leiter der Privaten Akademie für erweiterte Tiermedizin in Bad Wildungen (D); Tierarzt in der Praxisgemeinschaft für Ganzheitliche Veterinärmedizin Vet \& Physio in Oberalm bei Salzburg (A); FTA (A) für Akupunktur und Neuraltherapie, ZB (D): Physikalische Medizin und Physiotherapie sowie Akupunktur; www.vierbeiner-rehazent rum.de; info@vierbeiner-rehazentrum.de; vetundphysio.net

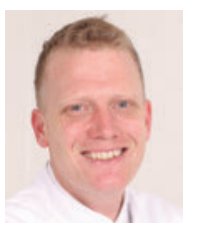

\section{Carsten Grußendorf}

Dr. med. vet.; Leitung Tiergesundheitszentrum Grußendorf, Zertifizierter Gutachter der GRSK (Gesellschaft für Röntgendiagnostik genetisch beeinflusster Skeletterkrankungen bei Kleintieren e.V.), Arbeitsschwerpunkte: Chirurgie, Orthopädie, Notfallmedizin, Zahnheilkunde, Ernährungsberatung

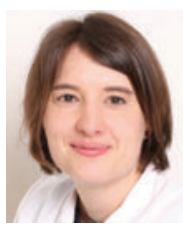

\section{Hanna Diekmann}

Dr. med. vet.; Promotion an der TiHo Hannover im Fachgebiet Orthopädie; bis 2019 Assistenztierärztin/Doktorandin in der Kleintierklinik des Tierärztlichen Instituts, Göttingen; seit 2019 im Tiergesundheitszentrum Grußendorf; Arbeitsschwerpunkte: Chirurgie, Orthopädie

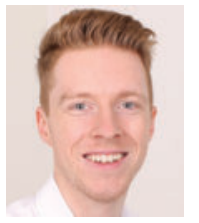

\section{Veit Kleine Büning}

Dr. med. vet.; 2017-2018 Internship im Tiergesundheitszentrum; seit 2018 im Tiergesundheitszentrum als Tierarzt

Literatur

[1] Kasper M, Zohmann A. Ganzheitliche Schmerztherapie für Hund und Katze. 2. Aufl. Stuttgart: Enke; 2011

[2] Alexander C. Physikalische Therapie für Kleintiere. 2. Aufl. Stuttgart: Enke; 2003

[3] Köppel E. Zur Entwicklung der Articulatio coxae und radiologischen Frühdiagnostik der Hüftgelenkdysplasie des Hundes. Eine morphologische Studie. Stuttgart: Enke; 1991

\section{Bibliografie}

DOI https://doi.org/10.1055/a-0961-5800

Hands on 2019; 1: 16-24

(c) Georg Thieme Verlag KG Stuttgart · New York ISSN 2628-6033 


\section{Refresher-Fragen: „Hüftgelenkdysplasie beim Hund“}

Beantworten Sie die folgenden Fragen und prüfen Sie direkt, ob Sie richtig liegen (s Seitenrand)! Mehrfachantworten sind möglich.

Viel Erfolg!

\section{Frage 1}

Im welchem Alter ist die knöcherne Ausbildung der Hüftpfanne abgeschlossen?
A 16. Lebenswoche
B 20. Lebenswoche
C 11. Lebensmonat
D $1 \frac{1}{2}$ Jahre
E 2 Jahre

\section{Frage 2}

Welche Muskeln stabilisieren das Hüftgelenk?
A M. pectineus
B M. semitendinosus
C M. biceps femoris
D M. gluteus medius
E M. iliopsoas

\section{Frage 3}

Ab welchem Alter sollte das horizontale Schwingen des Beckens (LSÜ-Twist) einem stabileren Schritt weichen?

A 16. Lebenswoche

B 20. Lebenswoche

C 6. Lebensmonat

D 12. Lebensmonat

E 24. Lebensmonat

\section{Frage 4}

Welche physiotherapeutischen Methoden eignen sich zur Kräftigung der hüftstabilisierenden Muskulatur?
A Thermotherapie
B Unterwasserlaufband
C $\mathrm{MOBI}$
D manuelle Lymphdrainage
E Lasertherapie

\section{Frage 5}

Auf welchem Prinzip basiert RITA?
A neurovegetativer Reflex
B polysynaptischer Reflex
C vasovagaler Reflex
D Massenreflex
E keine der Antworten ist richtig

\section{Frage 6}

Was versteht man unter Weichteil-HD?

A unvollständige Ausbildung des Pfannendachs

B Hypertonus des $M$. pectineus

C Laxizität der Gelenkkapsel

D Hypotonus des M. biceps femoris

E lockere Hüfe

\section{Frage 7}

Welche Aussagen zum Training mit Gewichtsmanschetten sind korrekt?

A Der Bewegungsablauf hat reflexartigen Charakter und läuft daher unkontrolliert ab.

B Eine Manschette oberhalb des Tarsus führt unter anderem zu einer Aktivierung der Glutealmuskulatur.

C Das Training ist für Welpen ab der 20. Lebenswoche empfehlenswert.

D Eine Manschette unterhalb des Tarsus führt unter anderem zu einer Aktivierung der Glutealmuskulatur.

E Das Training eignet sich nicht für Hunde, die unter einer HD leiden.

\section{Frage 8}

Welche physiotherapeutischen und physikalischen Methoden können in den meisten Fällen am 1. postoperativen Tag genutzt werden?
A manuelle Lymphdrainage
B reflexinduziertes Training nach Alexander
C Interferenzstrom-Regulationstherapie
D Tapping
E aktiv-assistive Methoden

\section{Frage 9}

Nach welcher OP-Methode ist es ausdrücklich erwünscht, dass sich der Hund nach der Operation frühzeitig intensiv bewegt?
A Totalendoprothese
B Femurkopfhalsresektion
C Pektinektomie
D TPO
E DPO 